\title{
Hollow Fiber Membrane Application for Blood Treatment
}

\author{
Akinori Sueoka* and Koichi Takakura \\ Medical Products Division, Kuraray Co., Ltd. \\ 1-12-39 Umeda, Kita-ku, Osaka 530, Japan
}

(Received December 10, 1990)

\begin{abstract}
Hollow fiber membranes have been widely used for extracorporeal blood treatment ranging from an artificial kidney, plasmapheresis to oxygenator. In particular, the artificial kidney is currently an established therapeutic modality for renal failure. Hitherto, various hollow fiber membranes have been developed and become commercially available, and thus in Japan alone about 15 million $-\mathrm{m}^{2}$ hollow fiber membranes have come to be used a year. In view of such a trend, many studies are being carried out to elucidate the interactions between the membrane and blood, such as antithrombogenicity or complement activation. In addition, modification of membranes is being investigated in order to prevent complications of long-term dialysis patients. The hollow fiber membrane is also employed in therapeutic plasmapheresis which aims to treat intractable diseases by removing pathogenic macromolecules in plasma. There are several clinical procedures which enable selective removal of harmful substances in blood with a combination of some membranes of different pore sizes. The membrane oxygenator consisting of microporous, hydrophobic hollow fibers is routinely used as a temporary alternative to the patient lung during open-heart surgery. The membrane method is considered to be superior to the conventional bubble type oxygenator because of less deleterious effects on blood components. Also, extracorporeal membrane oxygenation has been developed as a long-term assist in the treatment of respiratory insufficiency patients.

KEY WORDS: Hollow Fiber Membrane / Artificial Kidney / Plasmapheresis / Oxygenator / Blood Treatment / Blood Compatibility
\end{abstract}

The extracorporeal blood treatment with use of membranes has been widely applied to artificial kidney, plasmapheresis, and oxygenator. These techniques of blood purification treatment are all designed to remove harmful substances in the blood or correct the blood composition through diffusion, filtration, separation, or gas transfer. Remarkable progress has been made in recent years, particularly in the field of artificial kidney treatment. Annual production of hemodialysis membrane in Japan has reached about 15 million square meters and the worldwide output is estimated to be approximately three to four times greater. In the early stage of dialyzer development, flat-plate or coil type modules 
using flat-sheet or tubular membranes predominated. However, with advance in the spinning technology of hollow fibers, hollow fiber or capillary membrane devices have become predominant, amounting to more than 98 percent of the artificial kideny. The hollow fiber dialyzer is characterized by its compactness, easy handling, high dialysis efficiency and safety to patients, and therefore, has gained entirely wide acceptance. In Table 1 are represented applications of various hollow fiber membranes for blood treatment.

When membranes are used in contact with blood, the biocompatibility of hollow fiber membranes as well as their mass transfer characteristics are of primary importance. This paper presents a review of the application of hollow fiber membranes in the blood treatment including artificial kidney, plasmapheresis and oxygenator, focusing on the current developments and problems as well as future trend, with special reference to the interaction between blood and membranes.

Table 1 Application of various hollow fiber membranes for blood treatment

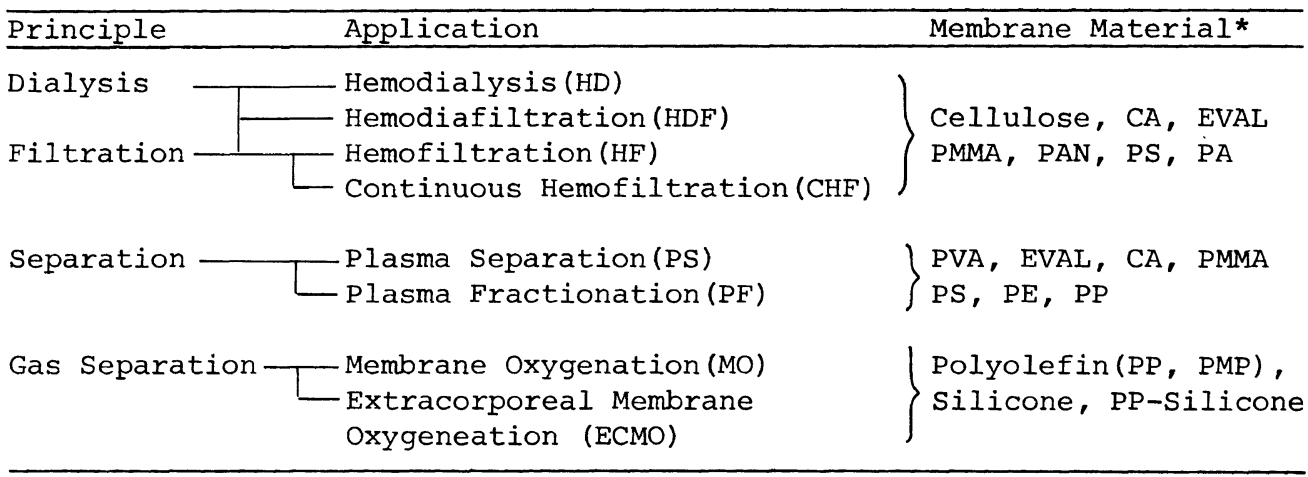

* CA; Cellulose acetate, EVAL; Ethylene-vinyl alcohol copolymer, PS; Polysulfone, PAN; Polyacrylonitrile, $\mathrm{PA}$; Polyamide, PE; Polyethylene, PP; Polypropylene, PMMA; Polymethyl methacrylate, PMP; Poly-4-methyl pentene-1

\section{MANUFACTURING METHOD AND STRUCTURE OF HOLLOW FIBER MEMBRANES}

Hollow fiber membranes are manufactured using various methods such as wet, dry, dry/wet and melt spinning processes, like textile fibers. The wet and dry/wet spinning methods are most often used because of easiness in controlling the pore structure and hollow-fiber profile. As a special membrane, microporous polyolefin hollow fibers are produced in the cold drawing process. The microporous membrane is also used as a substrate for a composite membrane.

There are several membrane structures: homogeneous, asymmetric and microporous. Each type of membrane is properly used depending upon the size of target substances to be removed from blood, and the treatment method to be applied.

\section{ARTIFICIAL KIDNEY}

1) Types of artificial kidneys and membranes

The artificial kidney is designed to remove excess amount of water 
and uremic toxins from the blood of the patient with renal failure. As is shown in Table 1 there are several methods now being carried out: hemodialysis(HD), hemofiltration(HF) and hemodiafiltration(HDF). The solute removal characteristics of these methods is shown in Figure $1 .^{1}$ HD offers a good efficiency of removing small molecules such as urea and creatinine, and has been widely accepted for the treatment of renal failure patients. HF shows excellent clearance of middle molecules, but low efficiency in removing small molecules and requires a large quantity of infusion fluid. HDF removes both small and middle molecules efficiently, and the application of HDF to a short-time dialysis is being investigated.

In terms of material, hollow fiber membranes are roughly divided into two categories: cellulosics and synthetic ploymers. Since the artificial kidney was first developed, cellulosic membranes have been most widely used in hemodialysis treatment

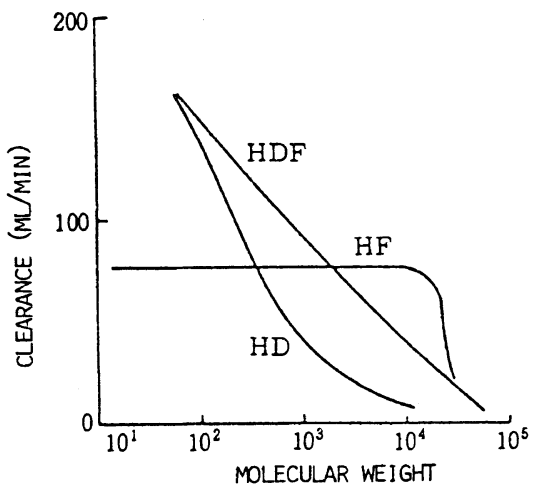

Fig. 1 Comparison of solute clearances by various methods ${ }^{1}$ up to now, mainly because of their high dialysis efficiency for small molecules, high mechanical strength and low production cost. On the other hand, in order to meet the needs of improving clearance of middle molecules and blood compatibility, a variety of synthetic polymer membranes have been developed and are gaining wider acceptance. In general, HD uses hydrophilic homogeneous dense membranes and both $\mathrm{HF}$ and $\mathrm{HDF}$ use hydrophobic asymmetric membranes.

2) Complications of long-term dialysis and high performance membrane

With advances in artificial kidney technology, such as improved performance of membrane, the number of dialysis patients in Japan has remarkably increased to 90,000 among which the long-term dialysis patients of over ten year history account for over 15 percent. As the population of long-term dialysis patients increases, complications including anemia, bone or joint disorder and peripheral neuropathy have developed to significant extent.

The reason for those complications has been explained as follows: HD using conventional membranes such as cellulose removes only small molecules including urea and creatinine, and thus middle and large molecules not removed gradually accumulate in the body, causing the development of various complications. However, it has been demonstrated that some dialysis complications such as anemia and bone pain have been successfully treated with HD using an EVAL membrane with a pore size large enough to allow the protein permeation to appreciable extent (protein permeating hemodialysis), and as the cause of anemia, the presence of an erythropoiesis inhibitor has been suggested in the middle and high molecular weight range. ${ }^{2}$

Recently, it has been reported that the accumulation of B2-microglobulin (MW 11,800) is the cause of carpal tunnel syndrome, observed for many long-term dialysis patients. ${ }^{3}$ This has prompted researchers to shift their target of removal from small molecules to middle molecules (MW 500-5,000) and further to low molecular weight proteins (MW 10,000- 
30,000). Following the EVAL membrane, high-performance membranes with high permeability to low molecular weight proteins have been successively developed using a number of synthetic polymers such as PMMA, polysulfone (PS) and PAN, and are now under clinical evaluation.

\section{3) Blood compatibility of hollow fiber membrane}

The safety of membranes and their biocompatibility with blood are important factors in the artificial kidney treatment. When the blood comes in contact with membrane, various reactions take place in the coagulation and immune systems. Recently, the interaction between membranes and blood has been extensively investigated. Antithrombogenicity of membranes and immune reactions including the complement activation are discussed as follows.

When the blood contacts with the membrane in the extracorporeal circulation, platelet and coagulation factors in blood are activated, causing blood coagulation. In ordinary hemodialysis treatments, an anticoagulant such as heparin is used to prevent this problem. But, a long-term use of heparin is said to develop some side effects such as abnomalities of lipid and bone metabolism and allergic reaction, and thus heparin is not allowed to use in immediate post-operative patients and those with bleeding risk factors.

In order to evaluate the antithrombogenicity of membrane materials, changes in the platelet and coagulation systems were determined by passing normal blood through minimodules incorporating different kinds of dialysis membranes, as illustrated in Figure 2.4,5 Figure 3 represents the influence of four kinds of membrane material on the platelet activating factors, platelet factor 4 (PF-4) and B-thromboglobulin $(B-T G)$. The platelet system was clearly activated by cellulose, PAN and PS membranes compared to EVAL membrane. The influence of membrane materials on the coagulation system was the same as the platelet system. These results indicate that the EVAL membrane has good antithrombogenic property. The antithrombogenicity of the EVAL membrane is reported to be ascribed to its unique molecular structure arising from copolymer of hydrophilic vinyl alcohol and hydrophobic ethylene, and the selective adsorption of albumin on the membrane surface. ${ }^{6}$ Because of it's antithrombogenic property the EVAL membrane allows the dialysis treatment without using any anticoagulant, and is currently being used for treating a variety of dialysis patients including those with a high bleeding risk factor.

In the meantime, extensive studies have been continuously carried out to improve the antithrombogenicity of other kinds of membrane materials. For example, polyethylene glycol (PEG) grafted cellulosic membrane exhibited good antithromogenicity. ${ }^{7}$ This can be explained in such a way that flexible hydrophilic PEG molecules on the membrane

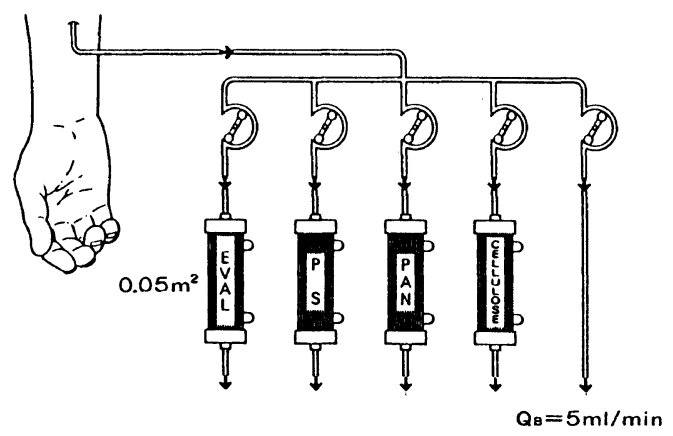

Fig. 2 Schematic diagram of ex vivo test circuit 4 
Hollow Fiber Membrane Application for Blood Treatment

surface form a

diffuse layer at

the blood-

material inter-

face so as to

prevent the pro-

tein and platelet

adhesion. It has

been reported

that a cellulose

membrane whose

surface is modi-

fied with phos-

pholipid polymer

has a structure

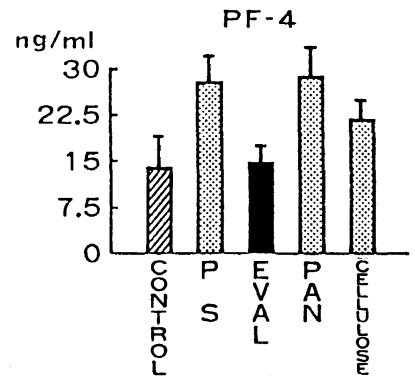

Fig. 3 The influence of four kinds of membrane materials on the platelet activating factors ${ }^{4}$

similar to the

biomembrane, showing good antithrombogenicity. 8 In addition, the development of antithrombogenic membranes is important to realize a wearable or an implantable type artificial kidney in future.

Transient leucopenia, a phenomenon of rapid decrease of white blood cell level observed about 15 to 30 minutes after the start of dialysis, has been reported by many investigators. 9,10 It has been clarified that it is caused by complement activation arising from the contact between the blood and the membrane.11 The degree of complement activation varies with the membrane material. Figure 4 shows changes in plasma concentrations of white blood cell and complement C3a during hemodialysis using cellulose membrane.12 In general the cellulosic membrane activates the complement system more significantly than synthetic polymer membranes. Many arguments have been made about the relations of complement activation with immuno deficiency in dialysis patients, and with acute symptoms such as allergic reaction, anaphylactic shock, itching, transient hypotension and hypoxia during dialysis. Although the relation between complement activation and clinical symptoms are not clarified yet, many efforts have been made to enhance the blood compatibility of membranes..$^{13}$ In cellulosic membranes, hydroxyl groups are considered to be responsible for complement activation, and thus some interesting improvements to prevent the complement activation have been made. These include the substitution of about $5 \%$ of hydroxyl groups of cellulose molecule by diethyl aminoethyl groups, ${ }^{14}$ and masking of the active hydroxyl groups on the membrane sur-

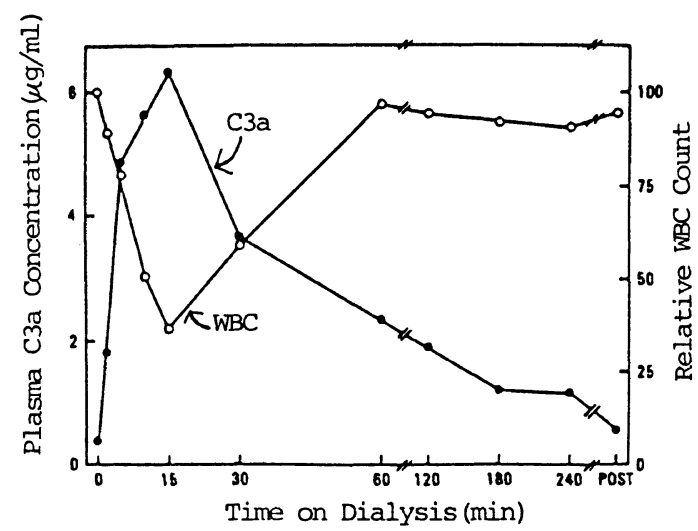

Fig. 4 Changes in plasma concentrations of complement $\mathrm{C} 3 \mathrm{a}$ and white blood cell during hemodialysis procedure using cellulose membrane 12

face by treating with cationic synthetic polymers containing amino groups, 15 and also the above-mentioned PEG grafted cellulose membrane. 
Some of them have now become commercially available as modified cellulose membranes, which show significant improvement in complement activation.

4) New developments

The glomerular basement membrane of the kidney are negatively charged, thus offering charge selectivity for solute removal. An attempt has been made to produce a negatively charged dialysis membrane by introducing carboxylic groups to the EVAL membrane. 16

Removal of water from the blood is investigated using the membrane distillation method, in which water is removed through the microporous PTFE membrane by utilizing a vapor pressure difference.17 It is argued that the membrane maintained its performance longer than other types of membrane because of less deposition of protein onto the membrane surface and that it could selectively remove water. Further study is under way.

Continuous arterio-venous hemofiltration(CAVH) has been developed and used for the treatment of acute renal failure.18 This method uses a hemofilter with a small membrane surface area to perform blood filtration continuously for several days, utilizing the arterio-venous pressure difference. Compared with the conventional intermittent treatment methods, $\mathrm{CAVH}$ is a therapeutic method similar to glomerular filtration and is expected to apply to the wearable type artificial kidney in future.

\section{PLASMAPHERESIS}

\section{1) Principle of membrane plasmapheresis}

Plasmapheresis is a blood processing method for all plasma components including large molecules with a molecular weight of a few million dalton, and is used for the treatment of various intractable diseases. The substances to be removed by plasmapheresis include: auto-antibodies and immune complexes in autoimmune diseases such as rheumatoid arthritis, systemic lupus erythematosus, or myasthenia gravis; hematological diseases such as macroglobulinemia; protein-bound substances in drug poisoning or fulminant hepatitis; and cholesterol in hyperlipidemia.

There are two types of plasmapheresis procedures available: plasma exchange which separates the patient blood into blood cell and plasma components with use of a plasma separator, replacing the plasma components with healthy human plasma; and plasma processing which selectively removes pathogenic substances from the separated plasma with the use of a membrane or adsorbent.

The plasma separator consists of microporous membranes with a pore size of 0.2 to $0.5 \mu \mathrm{m}$ to separate all blood cellular components $(2-3 \mu \mathrm{m})$ and plasma components. At present, there are many kinds of hollow fiber membranes available in the market for plasma separation: hydrophilic PVA, hydrophobic cellulose acetate (CA), PP, PE, PMMA, and PS. These membranes reject all blood cellular components, while allowing over 90 percent passage of large molecules such as albumin, IgG and IgM, respectively, thus it is possible to attain nearly perfect separation of blood cells and plasma.

The plasma exchange is a simple and efficient method, but it requires a large quantity of expensive replacement fluid (fresh frozen plasma or albumin preparations) and also there is possible risk of infection such as hepatitis due to the replacement fluid. To solve these problems, the double filtration or cascade filtration has been developed. As shown in Figure $5^{19}$ this method uses two kinds of filters with 
different pore sizes -- a plasma separator (primary filter) and a plasma fractionator (secondary filter) -- to selectively remove large molecules including pathogenic substances from plasma.

The plasma frac-

tionation membrane

has a pore size from

0.01 through $0.05 \mu \mathrm{m}$

and EVAL, CA or PE

hollow fiber mem-

brane is used for

this purpose. With

EVAL membranes, the

following three

types with different pore sizes are available; $2 \mathrm{~A}, 3 \mathrm{~A}$ and 4A. 20 Figure 6 shows the sieving coefficients of each EVAL membrane for plasma solutes.20 The 4A membrane is suitable for removing IgM (MW 950,000) fraction and $B-1$ ipoprotein

(MW 2,000,000). This membrane showed good sieving of albumin (MW 67,000) and removed an enough amount of harmful substances as well. To separate IgG (MW 160,000) fraction from albumin, the $2 \mathrm{~A}$ membrane seems to be suitable.

As a different approach to the plasma fractionation, the method, called cryofiltration, 21 has been developed to aim at selective removal of solutes by cooling withdrawn plasma at $4^{\circ} \mathrm{C}$ to form cryogel, followed by filtration with the secondary membrane with a pore size larger than that of the secondary membrane of the above-mentioned double filtration. It has been reported that cryofiltration can remove such pathogenic substances as auto-antibodies and immune complexes more selectively.

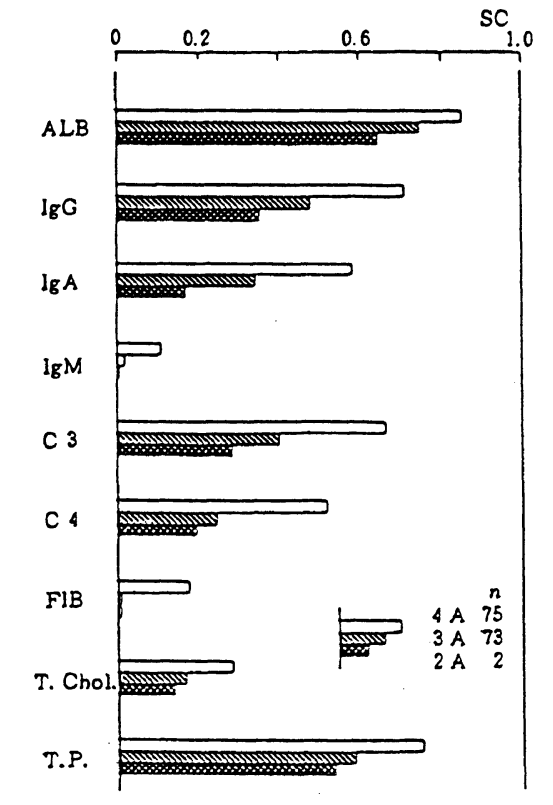

Fig. 6 Sieving coefficients of three types of EVAL plasma fractionators for various solutes ${ }^{20}$

2) Blood compatibility of membranes

The pores on the membrane surface are often clogged with protein deposits. This virtually means a reduced membrane area, leading to 
considerable reduction in filtration performance. Of various plasma separation membranes, the PVA membrane keeps stable filtration performance throughout the procedure, with less protein deposits on its surface, because of its hydrophilic nature. ${ }^{22}$

The sieving coefficients of EVAL and PE plasma fractionation membranes for solutes such as albumin, IgG, and fibrinogen are almost the same, indicating that the EVAL and PE membranes have almost the same pore size. However, as for the sieving of HDL cholesterol, there is a great difference between the two membranes, because PE membrane has high affinity to cholesterol resulting in the marked deposition of cholesterol on its surface. ${ }^{23}$ Therefore, when selecting a separation membrane, the affinity of the membrane to blood components should be taken into account as well as its pore size.

The complement activation of plasma separation membranes has also been investigated.24,25 From the studies on the changes of activated complement C3a level in patient bloods during plasma exchange using different kinds of membrane plasma separators, PVA and CA membranes have been reported to strongly activate the complement system, suggesting the close relation between hydroxyl groups on the membrane surface and complement activation. It is also pointed out that the complement activation is associated with the membrane structure as well as the material, because PMMA and CA plasma separation membranes activate the complement system to higher extent, compared to their dialysis membranes, as evidenced clearly by formation of high concentrations of activated complement $\mathrm{C} 3 \mathrm{a}$ in the plasma filtrate.

In addition to the complement activation, attention has been paid to the influence of membrane materials on the immune response and its importance in immunomodulation has been demonstrated. The effect of serum-membrane material interaction on lymphocyte response has been briefly studied for PVA and EVAL membranes using the in vitro perfusion model, since the lymphocyte plays a major role for the modulation of the immune system. ${ }^{26}$ The serum which had contacted the PVA membrane showed marked lymphocyte suppressive response, contrary to the EVAL membrane. This suggests that the PVA membrane separator may show beneficial immunosuppressive effect on the extracorporeal treatment of autoimmune diseases arising from highly activated immune system. Further study is now in progress.

\section{OXYGENATOR}

1) Principle of mem-
$\frac{\text { brane oxygenator }}{\text { The oxygenator is a }}$
device of artificial
lung which oxygenates
venous blood extracorpo-
really during open-heart
surgery. It is roughly
divided into two types:
bubble and membrane
types. The bubble oxy-
genator performs the
exchange of gases by
direct contact of blood
with oxygen, while in
the membrane type, the

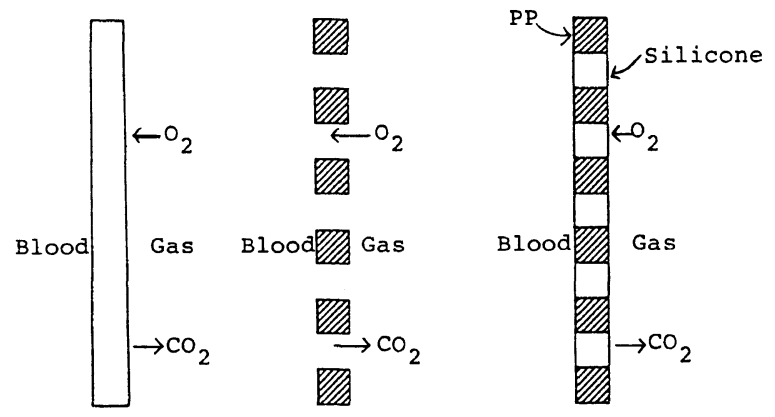
Dense
Membrane
(Silicone)
Porous
Membrane
(PP)
Composite
Membrane
(PP-Silicone)

Fig. 7 Membrane structure and gas transfer mechanism $^{27}$ 
gas exchange takes place through the gas-permeable membrane. The bubble oxygenator has been preferably used for a long period of time, however it is being displaced by the membrane type in recent years mainly because of problems of the erythrocyte destruction and protein denaturation. Today a number of gas-permeable membranes have become available. Most oxygenator membranes are a hollow fiber type. They fall roughly into the following two categories, as shown in Figure $7: 27$ homogenous membrane, for example, silicone membrane, which allows gas transfer by solubility and diffusion; and microporous polyolefin membrane such as PP membrane, which allows gas transfer through its micropores. The silicone material is highly gas-permeable, but has poor mechanical strength. On the other hand, the microporous PP membrane offers a high gas permeability but has a shortcoming of serum leakage during a long-term use.

\section{2) New developments}

Like the hollow fiber hemodialyzer, the oxygenator has so far adopted the internal perfusion method, in which the blood flows inside hollow fibers. ${ }^{28}$ Recently, however, the external perfusion method, which allows the blood to flow outside the hollow fibers, has come into wide use. In the new method, the blood flows at a right angle to the hollow fiber in such a way to reduce the boundary layer resistance at the blood side, thus increasing an efficiency of gas exchange. To attain a better performance of the oxygenator, the module design, such as the configuration of the hollow fiber bundle or the arrangement of hollow fibers inside the device, is also a key factor. For this purpose, a number of modules have been devised so far. For example, a laminated module of hollow fiber sheet has been made by knitting hollow fibers with fine threads to control the clearance between the hollow fibers. ${ }^{29}$ Because of such advantages as a good gas exchange performance and less chance of causing damage to the blood components, the external perfusion oxygenator has become predominant.

The microporous membranes work well when used for ordinary open-heart surgery ( 2 to 3 hours), but if used for a long period of time cause serum leakage due to the protein deposition on the surface, with a resultant rapid reduction in gas exchange efficiency. To prevent this problem, its micropores are filled with silicone or fluoropolymer, or the whole membrane surface is thinly coated with such polymers to form the composite membrane as shown in Figure 7. A microporous membrane so treated is free from serum leakage and offers a better gas permeability compared to the ordinary silicone membrane.

Recently, a hollow

fiber membrane with an asymmetric structure has been successfully manufactured by melt spinning under properly controlled drawing conditions by using poly-4-methylpentene-1 (PMP), as illustrated in Figure 8.29 The membrane has a skin layer on the outer surface of the sponge structure and thus is suitable for external perfusion. The
Process $\quad$ Spinning $\Longrightarrow$ Drawing $\Rightarrow$ Heat set

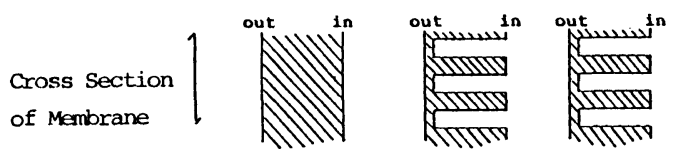

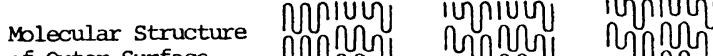

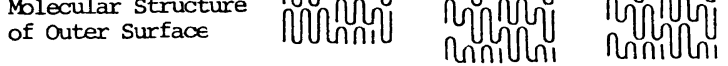

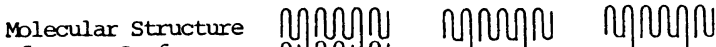
of Inner Surface

กuovol

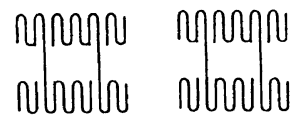

Fig. 8 Manufacturing process of poly-4methyl pentene-1 membrane with asymmetric structure 29 
skin layer is so thin, less than $1 \mu \mathrm{m}$, that it is highly permeable to gases and causes no serum leakage if used for a long-term oxygenation.

For the purpose of a long-term assist in the treatment of respiratory failure patients, the extracorporeal membrane oxygenation (ECMO) has been developed and is now in clinical use. Since the ECMO device is used for a certain period of time until the patient lung recovers normal function, the membrane is required to cause no serum leakage and to have good blood compatibility. Therefore, the PMP membrane with asymmetric structure and PP-silicone composite membrane are suitable for this method.

In addition, a new device, called an intravascular oxygenator (IVOX), has recently been developed and is in clinical trials. 30 It is interesting that IVOX utilizes the concept of intravenous oxygen delivery with the use of microporous PP hollow fibers, mimicking the natural exchange of gases in the blood.

\section{CONCLUSIONS}

In addition to the above-mentioned applications, the hollow fiber membrane has also been applied in such fields as the ascites treatment, hemoconcentration for autologous blood recovery, donor plasmapheresis and removal of virus in blood. It is noteworthy that remarkable progress has been made up to now in the application of membrane to the medical field involving blood treatment, and the blood treatment membranes occupy a major position in the market, since about 15 million-m ${ }^{2}$ artificial kidney membranes have come to be used a year in Japan.

At present, membrane separation of blood and plasma is attained mainly by its pore size. To achieve the higher selectivity, the development of a membrane with charge or adsorption function as well as uniform pore size is desired. With respect to blood compatibility, any sufficiently blood-compatible membrane has not been available so far. Most of the blood treatment membranes currently in use are made of general-purpose polymers developed mainly for industrial uses. Therefore, it is desirable to develop a tailor-made, biocompatible membrane with high performance and selectivity according to the purpose of use. It is expected that the application of membrane to the biomedical field including blood treatment will be further broadened in the future.

\section{REFERENCES}

1. K. Sakai and M. Mineshima, Kagaku-Kogaku, 45, 158, (1981)

2. A. Saito, H. Naito, M. Hirohata, Progress in Artificial Organs, 412 (1981)

3. F. Gejyo, T. Yamada, S. Odani, Biophys. Res. Commun., 129, 701 (1985)

4. H. Naito, T. Miyazaki, T. Takagi, A. Sueoka, S. Takashima, A. Kubotsu, Y. Inukai, Jpn. J. Artif. Organs, 16(2), 763(1987)

5. A. Sueoka and H. Naito, the 7th World Congress of the International society for Artificial Organs, Sapporo, Oct. 1-4, 1989

6. H. Naito, T. Miyazaki, A. Kubotsu, S. Takashima, K. Takakura, M. Kawahashi, Jpn. J. Artif. Organs, 14(1), 45 (1985)

7. K. Kino, K. Iizuka, K. Shishido, E. Kinugasa, T. Akizawa, T. Kitaoka, S. Koshikawa, A. Kishida, Y. Ikada, Jpn. J. Artif. Organs, $19(2), 731(1990)$

8. K. Ishihara, T. Ueda, N. Nakabayashi, Polymer J., 22(5), 355 (1990)

9. Y. Mito, A. Nishimura, A. Sumiyoshi, M. Kawai, Y. Nose, Y. Kawamura, C. Yoshimoto, Sogoigaku, 17, 86 (1960) 
10. L.S. Kaplow, J.A. Goffinet, JAMA, 203, 1135 (1968)

11. P.R. Craddock, J. Fehr, A.P. Dalmasso, K.L. Brigham, H.S. Jacob, J. Clin. Invest., 59, 879 (1977)

12. D.E. Chenoweth, "Hypersensitivity in Hemodialysis". ISAO Press, Cleveland, 1984, p11.

13. T. Akizawa, Jpn. J. of Clinical Diaysis, 4(7), 1009 (1988)

14. T. Bosch, Clin. Nephrol, 26 (Suppl. 1), 22(1986)

15. T. Akizawa, T. Kitaoka, S. Koshikawa, T. Watanabe, K. Imamura, T. Tsurumi, Y. Suma, S. Eiga, Trans. Am. Soc. Artif. Intern. Organs, $\underline{32}, 76(1986)$

16. A. Saito, H. Ogawa, T. Takagi, K. Ohta, H. Akasu, S. Kawai, A. Kubotsu, Trans. Am. Soc. Artif. Inter. Organs, 29, 673 (1983)

17. K. Sakai, T. Muroi, K. Ozawa, S. Takesawa, M. Tamura, T. Nakane, Trans. Am. Soc. Artif. Intern. Organs, 32, 397 (1986)

18. P. Kramer, J. Bohler, A. Kehr, H. Grone, J. Schrader, D. Mattaei, F. Scheler, Trans. Am. Soc. Artif. Intern. Organs, 28, 28(1982)

19. T. Agishi, H. Amemiya, K. Ota, N. Sugino, "Plasmapheresis. New Trends in Therapeutic Application". ISAO Press, Cleveland, 1983, p127.

20. A. Sueoka, T. Miyahara, K. Takakura, Y. Ueda, Jpn. J. Artif. Organs, $14(1), 413(1985)$

21. P.S. Malchesky, Y. Asanuma, I. Zawicki, M. Blumenstein, L. Calabrese, A. Kyo, R. Krakaur, Y. Nose, Artif. Organs, 4, 205 (1980)

22. P.S. Malchesky, J. Wojcicki, T. Horiuchi, JM. Lee, Y. Nose, "Plasmapheresis. New Trends in Therapeutic Applications". ISAO Press. Cleveland, 1983, p51.

23. Y. Takeyama, T. Horiuchi, M. Yamashita, PS. Malchesky, Y. Nose, Trans. Am. Soc. Artif. Intern. Organs, 33, 366 (1987)

24. T. Teraoka, J. Goldcamp, Y. Abe, T. Matsugane, E. Blasutig, J. Smith, P. Malchesky, Y. Nose, Trans. Am. Soc. Artif. Intern. Organs, $\underline{30}, 347$ (1984)

25. K. Takayama, T. Taira, Y. Nakashima, K. Tamura, E. Kinugasa, T. Akizawa, T. Kitaoka, S. Koshikawa, Jpn. Artif. Organs, 14(1), 344 (1985)

26. P.S. Malchesky and Y. Nose, Seminars in Hematology, 26(2), Suppl. 1 (April), 42 (1989)

27. F. Nomura, H. Hirose, H. Matsuda, S. Nakao, T. Sakakibara, M. Kaneko, Y. Sasako, Y. Miyamoto, K. Kadoba, S. Ohtake, T. Miura, E. Covino, Y. Kawashima, Jpn. J. Artif. Organs, 14(3), 1645 (1985)

28. J. Kamo, E. Hamano, I. Santo, A. Nakajima, K. Kamada, T. Yoshihara, Y. Fukui, T. Kawamura, Jpn. J. Artif. Organs, 14(3), 1637 (1985)

29. H. Akasu and T. Anazawa, J. Jpn. Soc. Biomaterials, 8(3), 141(1990)

30. J.D. Mortensen and G. Berry, Int. J. Artif. Organs, $\overline{12(6)}, 384$ (1989) 